\title{
Fluid extravasation caused by bladder perforation during bipolar transurethral resection using saline solution
} -a case report-

\author{
Sun-Kyung Park, Woo-Jin Cho, and Yun-Suk Choi \\ Department of Anesthesiology and Pain Medicine, Jeju National University Hospital, Jeju, Korea
}

Transurethral resection of the prostate is the gold standard in the operative management of benign prostatic hyperplasia. Bipolar transurethral resection of the prostate using saline solution is a safe technique and reduces the risk of transurethral resection syndrome. This report discusses a rare complication of bipolar transurethral resection of the prostate: the extravasation of $0.9 \%$ saline solution into intraperitoneal and retroperitoneal cavities due to bladder perforation, resulting in respiratory difficulties. (Korean J Anesthesiol 2013; 65: 163-166)

Key Words: Extravasation, Respiratory difficulties, Transurethral resection of the prostate.

Transurethral Resection of the Prostate (TURP) is commonly used to treat benign prostatic hyperplasia, and Transurethral Resection Syndrome (TURS) caused by nonconductive fluids (glycine, sorbitol, mannitol) which were used as irrigation fluid has been known as serious complications. Cases of TURS have been reported several times [1-3]. A variety of ways to reduce TURS have been suggested. A bipolar TURP that uses $0.9 \%$ saline solution as irrigation fluid lowers the risk of TURS and has recently come into wide use $[4,5]$. The $0.9 \%$ saline solution, a typical isotonic solution, is crystalloid fluid that has been used for more than 50 years in surgery, resuscitation, and maintenance [6].

What occurred with this case was extravasation of $0.9 \%$ saline solution into the intraperitoneal cavity due to perforation of the bladder while bipolar TURP was performed for benign prostatic hyperplasia, resulting in severe respiratory difficulties in the post anesthetic care unit (PACU). The associated pathophysiology, diagnosis, and treatment are discussed.

\section{Case Report}

An 84-year-old male patient, $165 \mathrm{~cm}$ in height and $84 \mathrm{~kg}$ in weight, visited the hospital to have TURP for hyperplasia of prostate with obstruction. The patient had no specific medical history. The preoperative chest X-ray and electrocardiogram (ECG) were within normal range. The hemoglobin level $(\mathrm{Hb})$ was $14.2 \mathrm{~g} / \mathrm{dl}$, and the hematocrit level was $41.1 \%$, which were within normal limits.

After arriving in the operating room, noninvasive blood pressure (NIBP), ECG, and pulse oximetry were attached. After a few minutes of rest, blood pressure was 150/95 mmHg, heart rate was 85 beats/min, respiratory rate was 15 breaths/

Received: October 12, 2012. Revised: 1st, November 29, 2012; 2nd, December 20, 2012. Accepted: December $22,2012$.

Corresponding author: Yun-Suk Choi, M.D., Department of Anesthesiology and Pain Medicine, Jeju National University Hospital, 66, Jejudaehak-no, Jeju 690-756, Korea. Tel: 82-64-717-2025, Fax: 82-64-717 -2042, E-mail: solafide5@yahoo.co.kr

(c) This is an open-access article distributed under the terms of the Creative Commons Attribution Non-Commercial License (http:// creativecommons.org/licenses/by-nc/3.0/), which permits unrestricted non-commercial use, distribution, and reproduction in any medium, provided the original work is properly cited. 
min, and oxygen saturation $\left(\mathrm{SaO}_{2}\right)$ was $94.0 \%$. The patient's position was maintained at the right lateral decubitus position. After identifying the L4-5 intervertebral space, a 25 G Quincke spinal needle was inserted into the space. After the stylet was removed and cerebrospinal fluid was confirmed to be draining well, $11 \mathrm{mg}$ of $0.5 \%$ bupivacaine (Marcaine ${ }^{\circledR}$, AstraZeneca AB, Södertälje, Sweden) with 100 ug of epinephrine was injected. Ten minutes after he was placed in the supine position, the height of sensory block was increased to the T12 and checked by pin-prick test. The patient was placed in a lithotomy position. When bipolar TURP began, the irrigation fluid with $0.9 \%$ saline solution was injected from a height of about $150 \mathrm{~cm}$ without pressure. The patient had a severely enlarged right lobe of the prostate. At 120 minutes after anesthesia, $4 \mathrm{mg}$ of ondansetron was injected because the patient vomited. At that time vital signs were stable (HR: 71 beats/min, NIBP: 150/85 mmHg). At 135 minutes after anesthesia, the patient complained of pain on operative site, considered to be caused by a low level of anesthesia. Therefore, with the consent of the patient, spinal anesthesia was switched to general anesthesia. Pre-oxygenation with $100 \%$ oxygen was conducted. After intravenous injection of $250 \mathrm{mg}$ of thiopental and $40 \mathrm{mg}$ of rocuronium, endotracheal intubation with a tube of internal diameter $7.5 \mathrm{~mm}$ was performed. Anesthesia was maintained with $2 \mathrm{~L} / \mathrm{min}$ of oxygen, $2 \mathrm{~L} / \mathrm{min}$ of air, and 2 vol\% of sevoflurane. Tidal volume was $500 \mathrm{ml}$ and respiratory rate was 10 breaths/min, so mechanical ventilation was initiated, when PIP was $24 \mathrm{cmH}_{2} \mathrm{O}$.

Forty minutes after switching to general anesthesia, the surgeon inserted a Foley catheter and finished the surgery. Glycopyrrolate $0.4 \mathrm{mg}$ and neostigmine $1.5 \mathrm{mg}$ were injected for reversal of muscle relaxation, the patient's spontaneous respiration and muscular strength recovered, and the endotracheal tube was removed. His blood pressure was well maintained at 140-100/100-70 $\mathrm{mmHg}$ throughout surgery, and the body temperature was around $36.2^{\circ} \mathrm{C}$. However during emergence $10 \mathrm{mg}$ of labetalol was injected twice due to high blood pressure $(180 / 110 \mathrm{mmHg})$. The total anesthetic time was 3 hours and 15 minutes, and the total surgical time was 2 hours and 30 minutes. Seven hundred fifty $\mathrm{ml}$ of Ringer's lactate were injected, and $5 \mathrm{~L}$ of $0.9 \%$ saline solution was used as an irrigation fluid. When the patient was transferred to the PACU, $\mathrm{SaO}_{2}$ was measured as $80 \%$. Although $10 \mathrm{~L}$ of $\mathrm{O}_{2}$ was provided with nonrebreathing reservoir facial mask, the $\mathrm{SaO}_{2}$ was not maintained at more than $90.0 \%$ (HR 77 beats $/ \mathrm{min}$, NIBP 190/110 mmHg). Arterial blood gas analysis showed a pH 7.159, $\mathrm{PaCO}_{2} 53.5 \mathrm{mmHg}, \mathrm{PaO}_{2} 58.9 \mathrm{mmHg}, \mathrm{HCO}_{3}{ }^{-} 15.9$ $\mathrm{mmol} / \mathrm{L}$, base excess $-9.0 \mathrm{mmol} / \mathrm{L}, \mathrm{Na}^{+} 139 \mathrm{mmol} / \mathrm{L}, \mathrm{K}^{+} 3.5$ $\mathrm{mmol} / \mathrm{L}, \mathrm{Hb} 14.2 \mathrm{~g} / \mathrm{dl}$ and $\mathrm{SaO}_{2} 81.8 \%$. The patient complained of severe respiratory difficulties and abdominal pain. On physical examination, abdominal distension was founded. The surgeon suggested conducting an abdominal CT scan, but oxygen saturation was not maintained at more than $90 \%$ despite an Ambu-bagging with $10 \mathrm{~L}$ of $100 \% \mathrm{O}_{2}$. The anesthesiologist decided to perform the endotracheal intubation again. Two hundred fifty mg of thiopental and $50 \mathrm{mg}$ of rocuronium were injected, and endotracheal intubation with a tube of ID $7.5 \mathrm{~mm}$ was performed. A chest X-ray was taken and an intravenous injection of $10 \mathrm{mg}$ of furosemide was conducted. In PACU NIBP was 180-140/110-85 mmHg and HR was $80-70$ beats/min.

A chest X-ray was normal. After the abdominal CT scan, the patient was transferred to the intensive care unit (ICU). Findings of the abdominal CT scan are seen Fig. 1. In the ICU, pressure support $11 \mathrm{cmH}_{2} \mathrm{O}, \mathrm{FIO}_{2}$ 0.45, and PEEP $6 \mathrm{cmH}_{2} \mathrm{O}$ were maintained as a PSV mode. Arterial blood gas analysis showed a $\mathrm{pH}$ 7.380, $\mathrm{PaCO}_{2} 26.1 \mathrm{mmHg}, \mathrm{PaO}_{2} 73.9 \mathrm{mmHg}$, $\mathrm{HCO}_{3}{ }^{-} 15.6 \mathrm{mmol} / \mathrm{L}$, base excess $-7.3 \mathrm{mmol} / \mathrm{L}$, and $\mathrm{SaO}_{2} 94.7 \%$. After the test, intravenous injections of $10 \mathrm{mg}$ of furosemide were injected twice. The total volume of urine on the day of the surgery was $1100 \mathrm{cc}$.

On the first day after surgery the endotracheal tube was removed and checked ABGA. Three hours later ultrasonography was conducted and showed that a great volume of fluid had accumulated around the liver. A percutaneous catheter drainage (PCD) was done into the right upper abdomen, and about $650 \mathrm{cc}$ of clear yellowish fluid was drawn. The patient was transferred to a general ward on that day. The PCD catheter was removed on the fourth day after the surgery. The patient was discharged from the hospital on the tenth day after the surgery.

\section{Discussion}

TURS is an iatrogenic form of water intoxication, a combi-

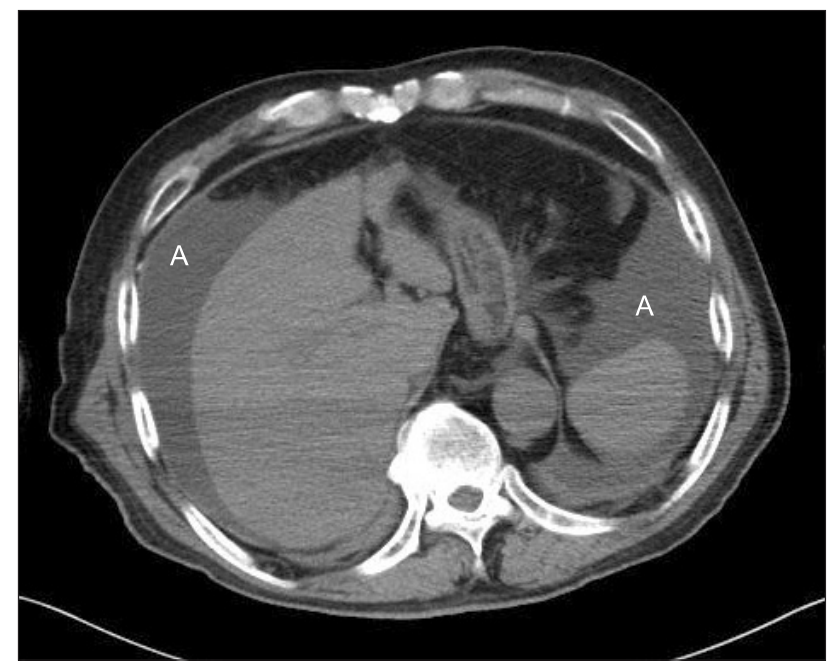

Fig. 1. CT scan of abdominal with patient supine. A large amount of ascites (A) can be visualized. 
nation of fluid overload and hyponatremia that is seen in a variety of endoscopic surgical procedures [1]. A recent largescale study indicated that the percentage of patients showing moderate clinical symptoms ranged to from 0.78 to $1.40 \%$ [1]. Many methods have been suggested to reduce risk of TURS, for example, reducing surgery duration, intraprostatic vasopressin injection, and cleansing with low pressure [1].

Currently bipolar TURP using $0.9 \%$ saline solution as an irrigation fluid is most often used: the solution to avoid TURS [2]. Bipolar TURP using $0.9 \%$ saline solution has a clinical effect similar to monoploar TURP using a nonconducting agent (glycerin) but it has lower bleeding risk, making it more commonly performed $[4,5]$. It rarely causes complications in TURP. As a bipolar electrosurgical tool incorporates the active pole and the return pole on the same electrode, $0.9 \%$ saline solution can be used as the conductive fluid medium. Therefore, hyponatremia caused by the use of nonconductive irrigation fluids (glycine, sorbitol, mannitol), CNS complications such as hyperglycemia and hyperammonemia that are related to glycine, and electrolyte imbalance have been reduced. There was no cardiovascular instability in this case, unlike other cases $[2,3]$. Infusion of excess $0.9 \%$ saline solution, however, temporarily causes dilutional hyperchloremic acidosis that raises the concentration of chloride a few hours after infusion and that lowers the base excess in a dose-dependent manner [6,7].

The patient in this case had serious acidosis in the PACU. Respiratory acidosis coincided with metabolic acidosis. After endotracheal intubation, bicarbonate and base excess did not change but $\mathrm{pH}$ recovered, proving that respiratory acidosis was a major problem. According to a study, infusion of $0.9 \%$ saline solution with colloid has less influence on acid-base [6]. Perforation of the bladder during TURP has been reported many times, but its incidence was only $0.7 \%[2,3,8]$. Patients with perforation usually complain of dyspnea, abdominal distension, and abdominal pain, major symptoms caused by extravasation of irrigation fluid into the intraperitoneal cavity $[2,3,8]$.

In this case, dyspnea was a major symptom, and perforation of the bladder was detected because of abdominal distension. The peritoneum, the biological membrane, allows electrolyte and fluid to pass through it depending on the components and the amount of extravasated fluid in the peritoneal cavity [2]. It has been reported that, besides respiratory difficulties and abdominal pain, hyponatremia was caused by perforation of the bladder when a nonconducting agent was used. In this case, however, hyponatremia was not seen because a $0.9 \%$ saline solution was used, and dyspnea was a major symptom since the accumulated $0.9 \%$ saline solution in the peritoneal cavity had a mass effect, resulting in an elevated diaphragm. Absorption of more than 3L of irrigation fluid brings about serious complications [7], but it is not easy to estimate how much irrigation fluid is absorbed.
Therefore, if irrigation fluid is mixed with $1 \%$ of glucose, it is possible to determine whether or not the irrigation fluid was absorbed by measuring the concentration of glucose in the blood within 30 minutes after surgery [9]. Fluid absorption through the open blood vessels frequently occurs not only during TURP but also arthroscopy and hysteroscopy that use irrigation fluid [10-15]. The biggest problem for the patient in this case was the accumulated fluid in the abdominal cavity, but it should be recognized that pulmonary edema can occur due to the volume overload of fluid absorption [7]. In this patient's case, the chest X-ray image was normal that was taken in PACU. The significant problem was that the accumulation of $0.9 \%$ saline solution in the abdominal cavity brought about dyspnea. Abdominal pain is strongly related to extravasation and is reported by $10-20 \%$ of patients who absorb $>1$ liter of fluid [10].

More than $20 \mathrm{mmHg}$ of intraperitoneal pressure caused by the accumulation of fluid in the abdominal cavity means abdominal compartment syndrome [10]. Fourteen to twenty $\mathrm{cmH}_{2} \mathrm{O}$ of intraperitoneal pressure causes renal and splenic dysfunction, and $34 \mathrm{cmH}_{2} \mathrm{O}$ of intraperitoneal pressure is an indication for emergent decompressive laparotomy [13]. Abdominal compartment syndrome can occur at any time when endoscopy using a fluid is performed. There is a possibility that the patient in this case, whose intraperitoneal pressure was not measured, had abdominal compartment syndrome, considering the elevated diaphragm as seen in the abdominal CT image. Signs and symptoms include abdominal pain, distension, decreased urine output, increased bladder pressure, increased peak inspiratory pressure, and decreased core temperature $[10,11,13]$.

For treatment, supportive care including diuretics is performed, and laparotomy can be performed when a patient is in a hemodynamically unstable condition. It was assumed that anesthesia had worn off when the patient in this case complained of pain at the operative site, but this could be regarded as abdominal pain. It was hard to discern other hemodynamic changes or abnormalities from a blood test at that moment. It is recommended, however, to suspect perforation of the bladder at an early stage and to check abdominal distension and dyspnea.

In conclusion, the patient in this case showed perforation of the bladder during bipolar TURP that resulted in respiratory difficulty due to the accumulation of $0.9 \%$ saline solution in the abdominal cavity, and he complained of abdominal distension and dyspnea, but was treated by endotracheal intubation for maintaining the airway, diuretics, and PCD.

\section{Acknowledgments}

This research was supported by the 2013 scientific promotion program funded by Jeju National University. 


\section{References}

1. Hawary A, Mukhtar K, Sinclair A, Pearce I. Transurethral resection of the prostate syndrome: almost gone but not forgotten. J Endourol 2009; 23: 2013-20.

2. Song JG, Kim YK, Kim YU, Seo HS, Kang KN, Hwang GS, et al. Transurethral resection syndrome occured by baldder perforation during transurethral resection. Korean J Anesthesiol 2007; 53: 254-8.

3. Dorotta I, Basali A, Ritchey M, O'Hara JF Jr, Sprung J. Transurethral resection syndrome after bladder perforation. Anesth Analg 2003; 97 : $1536-8$.

4. Mamoulakis C, Trompetter M, de la Rosette J. Bipolar transurethral resection of the prostate: the 'golden standard' reclaims its leading position. Curr Opin Urol 2009; 19: 26-32.

5. Michielsen DP, Coomans D, Braeckman JG, Umbrain V. Bipolar transurethral resection in saline: the solution to avoid hyponatraemia and transurethral resection syndrome. Scand J Urol Nephrol 2010; 44: 228-35.

6. Guidet B, Soni N, Della Rocca G, Kozek S, Vallet B, Annane D, et al. A balanced view of balanced solutions. Crit Care 2010; 14: 325.

7. Hahn RG. Fluid absorption in endoscopic surgery. Br J Anaesth 2006; 96: 8-20.

8. Kim SH, Shin WJ, Park JY, Kim YK, Hwang GS, Hwang JH. Pneumomediastinum due to inadvertent bladder perforation during transurethral resection of the prostate: A case report. Korean J Anesthesiol 2009; 56: 597-600.

9. Piros D, Fagerstrom T, Collins JW, Hahn RG. Glucose as a marker of fluid absorption in bipolar transurethral surgery. Anesth Analg 2009; 109: 1850-5.

10. Fowler J, Owens BD. Abdominal compartment syndrome after hip arthroscopy. Arthroscopy 2010; 26: 128-30.

11. Ladner B, Nester K, Cascio B. Abdominal fluid extravasation during hip arthroscopy. Arthroscopy 2010; 26: 131-5.

12. Lee GY, Han JI, Heo HJ. Severe hypocalcemia caused by absorption of sorbitol-mannitol solution during hysteroscopy. J Korean Med Sci 2009; 24: 532-4.

13. Sharma A, Sachdev H, Gomillion M. Abdominal compartment syndrome during hip arthroscopy. Anaesthesia 2009; 64: 567-9.

14. Verma M, Sekiya JK. Intrathoracic fluid extravasation after hip arthroscopy. Arthroscopy 2010; 26(9 Suppl): S90-4.

15. Woo YC, Kang H, Cha SM, Jung YH, Kim JY, Koo GH, et al. Severe intraoperative hyponatremia associated with the absorption of irrigation fluid during hysteroscopic myomectomy: a case report. J Clin Anesth 2011; 23: 649-52. 